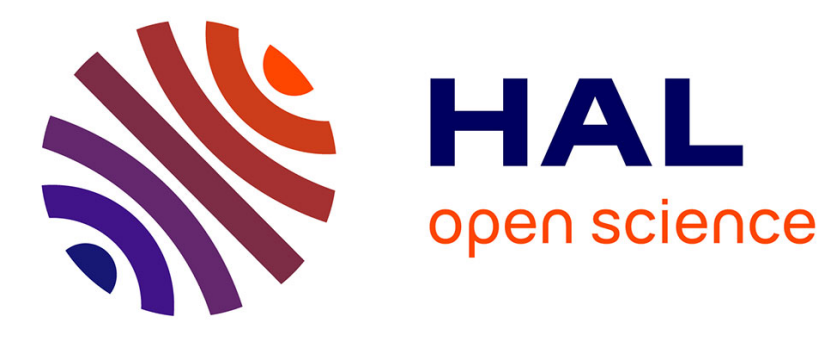

\title{
Oscillating Viscosity in a Lyotropic Lamellar Phase under Shear Flow
}

Anne-Sophie Wunenburger, Annie Colin, Jacques Leng, Alain Arnéodo, Didier

Roux

\section{- To cite this version:}

Anne-Sophie Wunenburger, Annie Colin, Jacques Leng, Alain Arnéodo, Didier Roux. Oscillating Viscosity in a Lyotropic Lamellar Phase under Shear Flow. Physical Review Letters, 2001, 86, pp.1374. 10.1103/PhysRevLett.86.1374 . hal-00097176

\section{HAL Id: hal-00097176 https://hal.science/hal-00097176}

Submitted on 21 Sep 2006

HAL is a multi-disciplinary open access archive for the deposit and dissemination of scientific research documents, whether they are published or not. The documents may come from teaching and research institutions in France or abroad, or from public or private research centers.
L'archive ouverte pluridisciplinaire HAL, est destinée au dépôt et à la diffusion de documents scientifiques de niveau recherche, publiés ou non, émanant des établissements d'enseignement et de recherche français ou étrangers, des laboratoires publics ou privés. 


\title{
Oscillating Viscosity in a Lyotropic Lamellar Phase under Shear Flow
}

\author{
A. S. Wunenburger, A. Colin, J. Leng, A. Arnéodo, and D. Roux \\ Centre de Recherche Paul Pascal, CNRS, Av. Dr. A. Schweitzer, 33600 Pessac, France
}

(Received 8 June 2000)

\begin{abstract}
We report some time-dependent behavior of lyotropic lamellar phase under shear flow. At fixed stress, near a layering instability, the system presents an oscillating shear rate. We build up a new stress versus shear rate diagram that includes temporal behavior. This diagram is made of two distinct branches of stationary states which correspond, respectively, to disordered and ordered multilamellar vesicle phases. When increasing the shear stress, prior to the transition to the ordered structural state, sustained oscillations of the viscosity are recorded. They correspond to periodic structural change of the entire sample between a disordered and a ordered state of multilamellar vesicles.
\end{abstract}

DOI: $10.1103 /$ PhysRevLett.86.1374

The behavior of complex fluids under shear flow has been extensively studied using rheology [1]. In these experiments, the fluid is generally sheared between a fixed and a moving plate. Controlling the velocity of the moving plate, the force exerted on the fluid is measured (shear rate controlled) or imposing a force, the displacement of the plate is recorded (shear stress controlled). The macroscopic shear rate $\dot{\gamma}$ is defined as the ratio of the velocity over the gap and the shear stress $\sigma$ is defined as the macroscopic force divided by the surface. Assuming a homogeneous response of the system, characteristic flow curves ( $\sigma$ as a function of $\dot{\gamma}$ ) are drawn. By comparison with a Newtonian behavior, a viscosity is defined through the relation $\sigma=\eta \dot{\gamma}$. Notice that this viscosity is thus extracted from two macroscopic quantities and is not easily related to the microscopic viscosity. For a non-Newtonian fluid, this macroscopic viscosity is a function of the shear rate $\eta(\dot{\gamma})$ or of the shear stress $\eta(\sigma)$, depending on the controlled parameter. In this paper, we will show that this macroscopic viscosity can display nonlinear behavior such as bistability and sustained temporal oscillations.

In addition to the fact that rheology characterizes a macroscopic response of the system, the interpretation of the rheological properties is made difficult by the coupling between structure and flow in complex fluids. In the past decade, the development of rheophysical tools (x-ray, neutron, and light scattering, but also dielectric measurements or NMR under shear) has allowed experimentalists to probe the fluid structure under flow [2]. From these structural studies under shear, we have learned that, in many systems, instabilities can develop at some critical shear rate or stress leading to a qualitative change of the fluid structure. These instabilities are different from classical hydrodynamic instabilities occurring in Newtonian fluids (i.e., Taylor instability) [3]. They involve mesoscopic scales (typically about a micron), they appear in homogeneous flow, and are thus characteristic of complex fluids. They may correspond to a phase transition existing at thermal equilibrium (such as the isotropic/nematic transition) [4-6] or to a textural change (nematics $[7,8]$ or smectics $[9,10])$. In the latter situation, the shear flow
PACS numbers: 83.10.Gr, 47.50.+d, 83.85.Cg

affects the density of defects and induces, in the case of lamellar phases, the formation of compact multilamellar vesicles [11]. Those shear effects may be summarized in a shear diagram that specifies the nature of the stationary states (texture or phase) observed for different shear rates (or shear stress). These states are separated by some dynamical transitions (i.e., the above-mentioned instabilities) and are most often described as a jump between two branches of steady states. Rheological experiments show that these transitions actually correspond to a discontinuity in the shear rate variable $[6,12-14]$ or in the stress variable $[12,15]$. However, as emphasized in previous papers [12], the fact that the complex fluid is sheared, and thus is maintained out of equilibrium, should lead to richer behavior than a simple jump between two stationary states. As for many other instabilities, we do expect, in certain cases, transitions to oscillating states and ultimately to chaotic dynamics. Very recently, it has been effectively proposed to use the theoretical framework of strange attractor to describe the experimental behavior observed in a living micelles system under shear flow [16]. In this Letter, we report the results on a lyotropic smectic system near what has been named a layering transition [17]. Sustained oscillations have been recorded and followed with both rheology and light scattering. The novelty of this work is to demonstrate that these large amplitude relaxation oscillations correspond to periodic structural changes of the entire sample between a disordered and an ordered state of multilamellar vesicles. We comment on the origin of those oscillations of viscosity and argue that they do not come from instabilities observed classically in Newtonian fluids.

Lyotropic lamellar phases have shown a richness of behavior under shear flow [2]. The texture of those systems is clearly modified by the flow. The spatial orientation of the lamellae changes as a function of the shear rate $[2,11]$. Figure 1 presents the shear diagram for a lamellar phase composed of sodium dodecyl sulfate (SDS), octanol, and brine [17]; this system shows several instabilities, among which is a transition between two states of onions (multilamellar spherulites) [11] labeled states II and IV, respectively. State II corresponds to a disordered state: the 


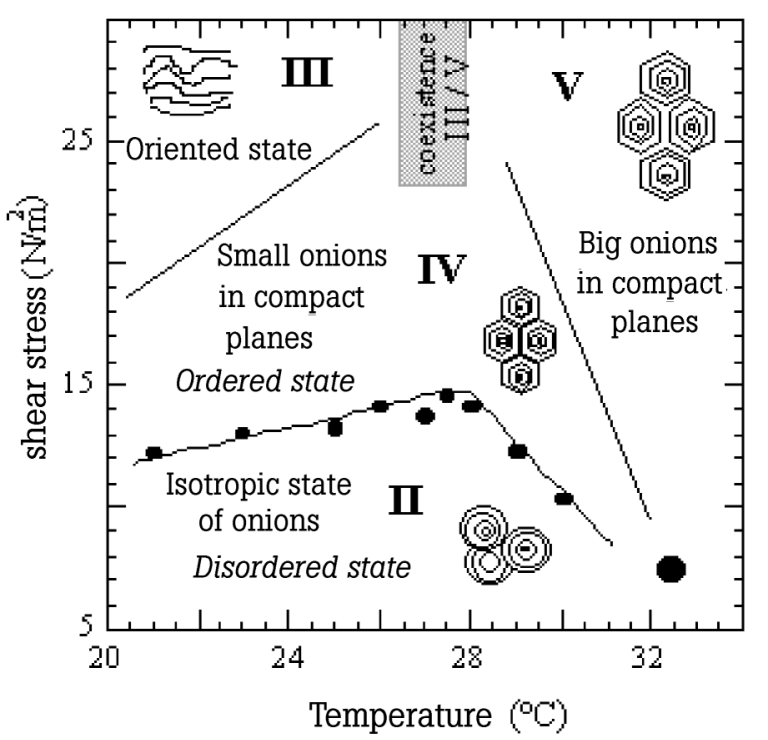

FIG. 1. Experimental shear diagram of a lamellar phase composed of SDS-octanol brine (weight fractions: 6.5\% of SDS, $7.8 \%$ of octanol, and $85.6 \%$ of water with $20 \mathrm{~g} / 1$ of $\mathrm{NaCl}$ ). This diagram shows the different orientations of the lamellar phase in a function of the shear stress $\sigma$ and the temperature. We mainly focus on the II-IV transition between a state of spatially noncorrelated onions (state II) and an ordered state (state IV), where onions are arranged in hexagonal planes.

spherulites do not exhibit long range order but only short range correlations such as in a liquid or a glassy state. State IV is an ordered state, where the onions are organized in hexagonal planes that flow over each other $[17,18]$. The difference between these two states can be easily characterized by using light scattering. Figure 2 shows the small angle light scattering patterns of states II and IV. The modulation of the ring of scattering in Fig. 2(b) (state IV), as compared to the isotropic ring of the disordered state in Fig. 2(a) (state II), is the signature of the long range correlations of the orientation of the hexagonal planes $[17,18]$. This transition can be characterized by using rheology. Taking into account only stationary states, the stress/shear rate curve exhibits a jump in shear rate above a critical stress value $\sigma_{c}$ [17] (Fig. 3). This value is a function of both the temperature and the exact composition of the sample. Significantly below $\sigma_{c}$, the system is in the disordered state II [Fig. 2(a)]; significantly above this critical value, the system is in state IV [Fig. 2(b)]. In fact, if one studies carefully the rheological behavior of this system, one notices that the critical shear stress is hard to define because of the appearance of time-dependent behavior in the neighborhood of the transition. In the following, we will pay particular attention to the temporal variations of the shear rate which imply temporal evolution of the viscosity.

We work on a sample corresponding to the following composition: $6.5 \%$ of SDS, $7.8 \%$ of octanol, and $85.6 \%$ of water with $20 \mathrm{~g} / 1$ of $\mathrm{NaCl}$ in weight fraction. The ex-

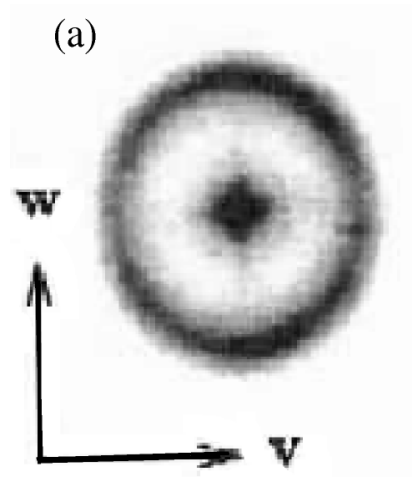

(b)

FIG. 2. Small angle light scattering patterns: (a) state II, (b) state IV.

periments are performed on a stress controlled rheometer (Carrimed CSL 100) using both a Mooney-type Couette and cone-plate cells. A homemade program is used to both control the approach and record the temporal evolution of the shear rate. As stated previously, the transition is very sensitive to both temperature and composition, including impurities. Consequently, for each new sample, the temperature has to be adapted to recover the transition at a given stress value. We impose a constant shear stress and record the shear rate as a function of time. The approach is done as adiabatically as possible in varying the shear stress by a very small amount and waiting for a long time (typically hours) between each data point. When increasing the shear stress, we observe the following

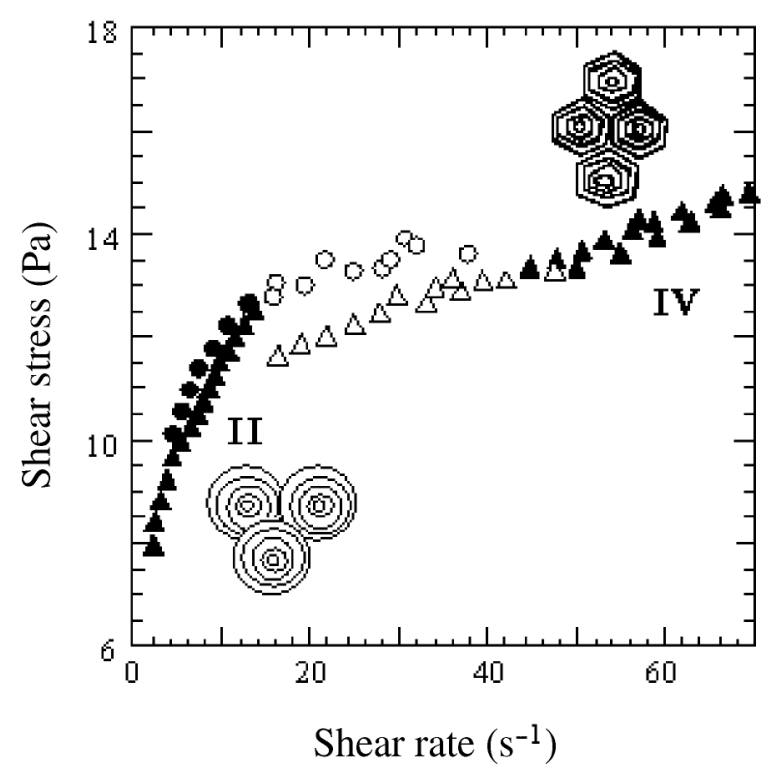

FIG. 3. Shear stress versus shear rate bifurcation diagram for the SDS-octanol-brine lamellar system in a cone-plate cell at $T=28^{\circ} \mathrm{C}$. The symbols have the following meaning: (black triangles) stable stationary state, (black circles) exponentially damped oscillations, (empty circles) sustained large amplitude relaxation oscillations, and (empty triangles) small amplitude noisy behavior. 


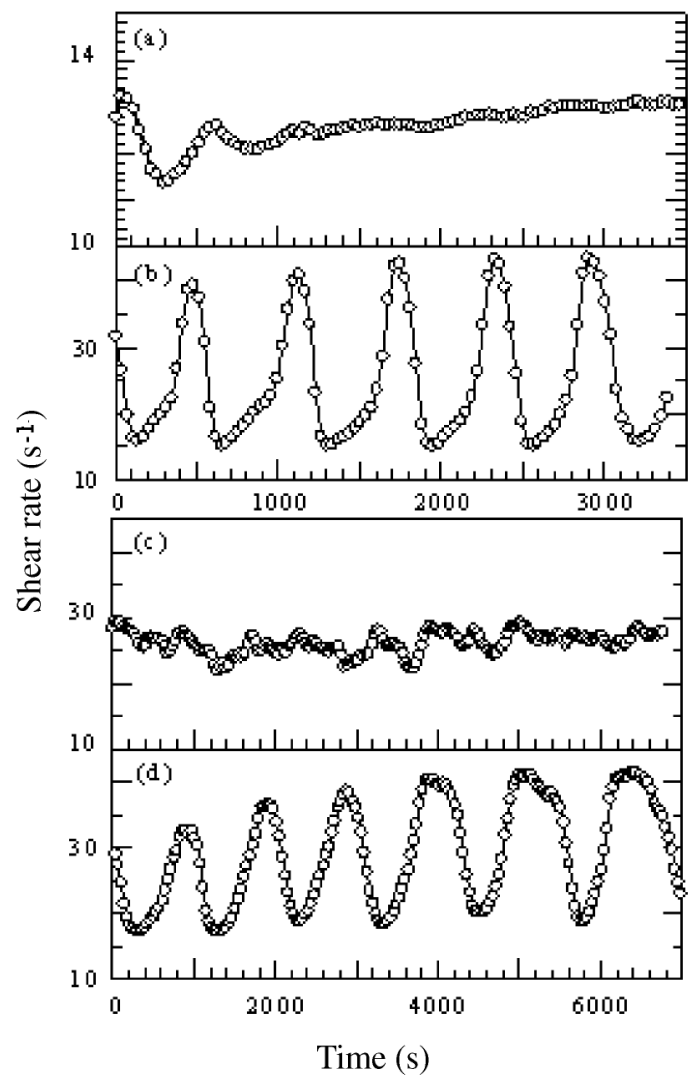

FIG. 4. Temporal behavior of the shear rate that can be observed for a SDS-octanol-brine lamellar phase submitted to a constant shear stress in a cone-plate cell at $T=28{ }^{\circ} \mathrm{C}$ : (a) damped oscillations, (b) sustained large amplitude relaxation oscillations, and (c) small amplitude noisy behavior. (d) Large amplitude oscillations observed in a Couette cell at $T=25^{\circ} \mathrm{C}$ are shown.

dynamical scenario near the transition II(disordered onions)-IV(ordered onions). Far enough from the critical shear stress, we observe an exponentially rapid relaxation of the shear rate towards its asymptotic steady state value. When the shear stress becomes closer to $\sigma_{c}$, we record some damped oscillations on the shear rate signal [Fig. 4(a)]. Then, if one keeps increasing $\sigma$, we witness the appearance of finite amplitude regular oscillations of the shear rate [Fig. 4(b)]. The period of these oscillations is rather large; for a given sample it can vary with the temperature from $700 \mathrm{~s}\left(T=28^{\circ} \mathrm{C}\right)$ at $2000 \mathrm{~s}$ $\left(T=22^{\circ} \mathrm{C}\right)$ (Fig. 5). The amplitude of these oscillations covers the whole range between the two steady branches corresponding to state II and state IV. In order to check whether or not these relaxation oscillations do correspond to structural oscillations, we probe rheology and light scattering patterns in parallel using a transparent Couette cell setup on the rheometer. Figure 6 shows a series of pictures taken as a function of time simultaneously to the recorded oscillations of Fig. 4(d) [19]. We clearly see that the minimum of shear rate corresponds to the so-called disordered state (state II) and the maximum of

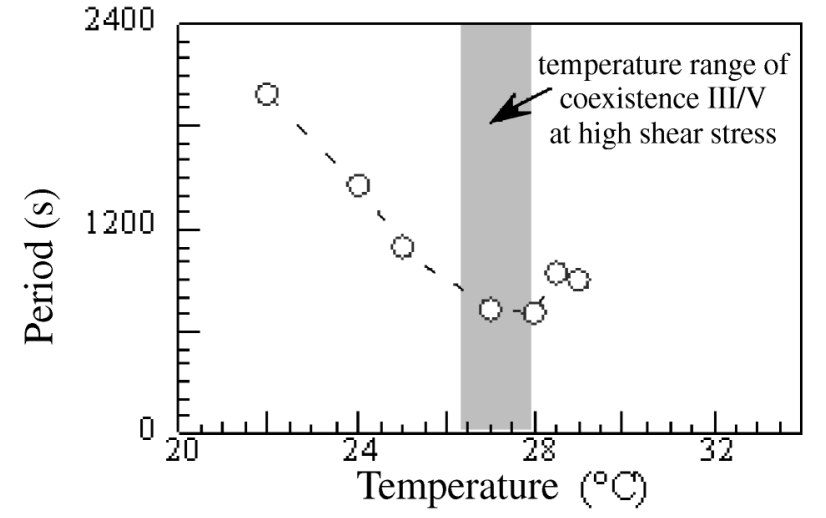

FIG. 5. Evolution of the period of the large amplitude relaxation oscillations as a function of the temperature.

the shear rate to the ordered state (state IV). In between, the modulation of the hexagonal pattern goes continuously from no contrast at all (state II) to a maximum of contrast (state IV). Looking at the entire cell in the vertical direction, no spatial inhomogeneities such as shear banding are observed during the oscillations (our setup does not allow us to investigate the possibility of a transient structuration within the gap). When further increasing $\sigma$, one ultimately reaches the steady state IV branch. But, as shown in Fig. 3, if one decides now to decrease $\sigma$ adiabatically, the system will asymptotically converges as a function of time to state IV and this even below $\sigma_{c}$. Indeed one observed some hysteresis phenomenon which

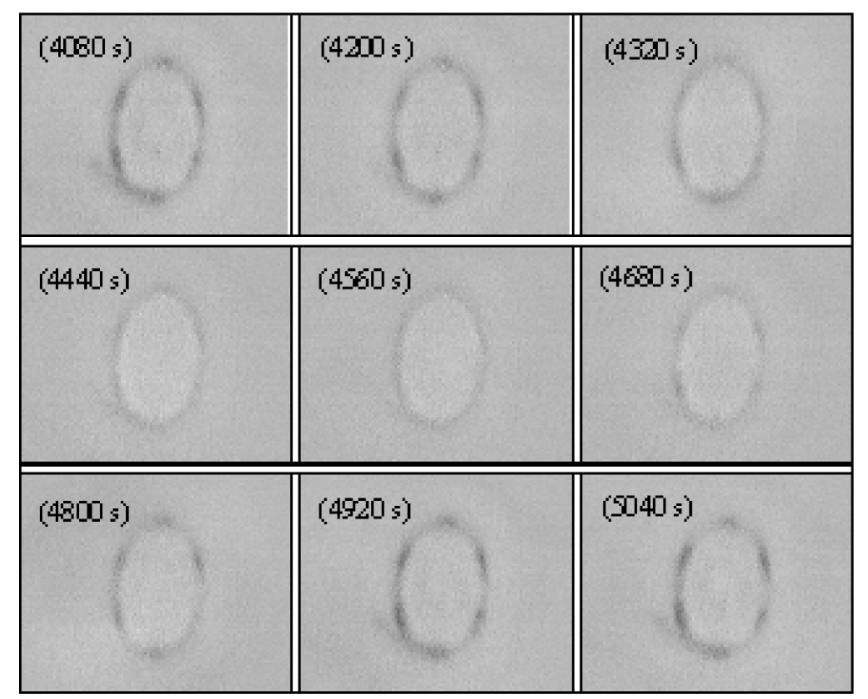

FIG. 6. Light scattering images recorded during the experiment shown in Fig. 4(d). Pictures are taken every two minutes. The lamellar phase is submitted to a constant shear stress $(\sigma=12.5 \mathrm{~Pa})$ at temperature $T=25^{\circ} \mathrm{C}$. The correlation peaks appear and disappear periodically in time, in phase with the periodic increases and decreases of the shear rate [Fig. 4(d)]. The light scattering pattern is somehow deformed by the optical setup. 
reveals the existence of a finite range of shear stress values over which both states II and IV coexist. This means that, for a given value of the shear stress, the system is bistable; it can be equally in two possible structural states of different viscosities.

The observation of oscillations in complex systems is not completely new; in recent works such behavior has been reported in dilute wormlike micelles solution [15] and in concentrated lamellar phase [20] under shear flow. The novelty of the present paper is that it brings the experimental demonstration that these oscillations of the macroscopic viscosity are clearly associated with two distinct structural steady states. They actually correspond to structural oscillations. The question persists, however, about the origin of these oscillations: Are they really due to the intrinsic physics of the transition or to a coupling with the mechanics of the rheometer? Since we could not rule out completely the last hypothesis, we checked many points so that we were as comfortable as possible with the first hypothesis. The time scale involved in the oscillations (typically $1000 \mathrm{~s}$ ) is far from the characteristic times involved in the experimental setup (at this shear rate, a complete rotation of the rotor is performed in a time of the order of $10 \mathrm{~s}$ ). Note that this time scale also excludes a simple stick-slip phenomenon. Moreover, we checked that this dynamical state does not depend upon the geometry we used or the inertia of the cell. Indeed, for a given temperature, we find the same behavior and the same period for the oscillations using a Couette cell or a cone plate. However, these periods are very sensitive to a small change of the temperature (a few degrees, as seen in Fig. 5) or of the composition of the sample. Consequently, we believe that these oscillations result from an out-of-equilibrium transition that can be described in the framework of bifurcation theory [21]. Let us mention that we also observe some apparently sustained oscillations when decreasing $\sigma$ from the ordered steady state IV. As shown in Fig. 4(c), these oscillations look very irregular and are of small amplitude. With our present apparatus and according to our experimental protocol, there is no way in this case to decide whether these oscillations actually correspond to deterministic chaos issued from the destabilization of state IV or to some unrelaxed noisy behavior about state IV on the verge of criticality.

In conclusion, we reported in this Letter the experimental observation of structural bistability and of sustained structural relaxation oscillations in a lyotropic smectic system under shear. As previously experienced in a different context, namely, nonlinear chemical reactors [22,23], one can hope to use dynamical systems theory to understand the evolution of the dynamics that is likely to result from the interaction of a hysteresis bifurcation (which accounts for the phenomenon of bistability) and a Hopf bifurcation (which underlies the propensity of viscosity to oscillate). In future work, we will use the normal form approach [21,23] as a guide for positioning our system in the parameter (e.g., temperature, composition) space, with the specific goal to identify and characterize the scenario leading to chaotic behavior of the macroscopic viscosity.

We are very grateful to R. Bernon and P. Merzeau for technical assistance and to J. Elezgaray for stimulating discussions.

[1] R. G. Larson, The Structure and Rheology of Complex Fluids (Oxford University Press, New York, 1999).

[2] D. Roux, in Theoretical Challenges in the Dynamics of Complex Fluids, edited by T. Mc Leish, NATO ASI Series E, Vol. 339 (Kluwer Academic, Dordrecht, 1997), p. 203; in Soft and Fragile Matter: Nonequilibrium Dynamics, Metastability and Flow, edited by M. E. Cates and R. Evans (Institute of Physics Publishing, Bristol, 2000), p. 185.

[3] J. Gollub and H. Swinney, Hydrodynamic Instabilities (Springer, Berlin, 1981).

[4] C. R. Safinya, E. B. Sirota, and R. J. Plano, Phys. Rev. Lett. 66, 1986 (1991).

[5] V. Schmitt, C. Marques, and F. Lequeux, Phys. Rev. E 52, 4009 (1995).

[6] J. F. Berret, D. C. Roux, and P. Lindner, Eur. Phys. J. B 5, 67 (1998)

[7] J. B. Riti et al., J. Rheol. 41, 1247 (1997).

[8] J. F. Berret, G. Porte, and J. C. Decruppe, Phys. Rev. E 55, 1668 (1997).

[9] C. R. Safinya et al., Science 261, 588 (1993).

[10] P. Panizza, P. Archambault, and D. Roux, J. Phys. II (France) 5, 303 (1995).

[11] O. Diat and D. Roux, J. Phys. II (France) 3, 1427 (1993).

[12] D. Roux, F. Nallet, and O. Diat, Europhys. Lett. 24, 53 (1993).

[13] C. Grand, J. Arrault, and M. E. Cates, J. Phys. II (France) 7, 1071 (1997).

[14] D. Bonn et al., Phys. Rev. E 58, 2115 (1998).

[15] Y. T. Hu, P. Boltenhagen, and D. J. Pine, J. Rheol. 42, 1185 (1998).

[16] R. Bandyopadhyay, G. Bassappa, and A. K. Sood, Phys. Rev. Lett. 84, 2022 (2000).

[17] P. Sierro and D. Roux, Phys. Rev. Lett. 78, 1496 (1997).

[18] O. Diat, D. Roux, and F. Nallet, Phys. Rev. E 51, 3296 (1995).

[19] Note that the ellipsoidal shape of the ring of scattering is an optical effect due to the small size of the Couette cell that acts as a cylindrical lens.

[20] C. Meyer, S. Asnacios, C. Bourgaux, and M. Kleman, Mol. Cryst. Liq. Cryst. 332, 531 (1999).

[21] J. Guckenheimer and P. Holmes, Nonlinear Oscillations, Dynamical Systems and Bifurcations of Vector Fields (Springer, Berlin, 1984).

[22] F. Argoul, A. Arneodo, P. Richetti, and J. C. Roux, J. Chem. Phys. 86, 3325 (1987).

[23] P. Richetti, J. C. Roux, F. Argoul, and A. Arneodo, J. Chem. Phys. 86, 3339 (1987). 